\title{
The development of a short food frequency questionnaire to assess diet quality in UK adolescents
}

\author{
$\underline{\text { Sarah Shaw }}^{1,2}$, Sarah Crozier $^{1}$, Sofia Strommer ${ }^{1,2}$, Hazel Inskip ${ }^{1,2}$, Mary Barker ${ }^{1,2}$ and \\ Christina Vogel ${ }^{1,2}$ \\ ${ }^{1}$ MRC Lifecourse Epidemiology Unit, University of Southampton, Southampton, United Kingdom and \\ ${ }^{2}$ NIHR Southampton Biomedical Research Centre, University of Southampton and University Hospital Southampton \\ NHS Foundation Trust, Southampton, United Kingdom
}

\section{Abstract}

UK adolescents have poorer diets than other age groups. Improving adolescents' diets has the potential to improve their health now and in later life, and the health of their future offspring. Established dietary assessment techniques can be difficult to use with adolescents due to high participant burdens. Robust and easy-to-implement techniques are required to assess adolescent diet in large-scale studies. This study aimed to identify the key indicator foods that contribute most to better quality dietary patterns in UK adolescents for use in a short food frequency questionnaire (FFQ).

Dietary data, collected using 4-day diet diaries, and nutritional biomarker data from waves 1-8 of the National Diet and Nutrition Survey rolling programme were used. Principal component analysis (PCA) was applied to 139 food groups to identify the key indicator foods that contribute most to better quality dietary patterns.

A 20-group diet score was calculated using coefficients for the 20 indicator foods from the 139-group PCA and multiplying by their standardised reported frequency of consumption. Scores were standardised to a mean of zero and a standard deviation (SD) of one.

The association and the agreement between the 139-group diet quality score and 20-group score were calculated using Spearman's correlation coefficient and Bland-Altman limits of agreement, respectively. Spearman's correlations were used to examine the associations between the two diet quality scores and nutritional biomarkers.

NDNS dietary data were available for 1282 boys and 1305 girls aged 11-18 years. The first PCA component explained 3.0\% of variance in the dietary data. A high-quality diet was characterised by greater consumption of fruit, vegetables, beans and pulses, wholegrains, nuts, and tap water, plus lower consumption of sugar-sweetened beverages, chips, processed meats, white bread, crisps, whole milk, baked beans, and added sugar.

A correlation of 0.86 was observed between the full 139 -group score and the 20 -group score with the difference between being 0 SDs. Bland-Altman 95\% limits of agreement were -0.98 to 0.98 SDs. Correlations, in the expected direction, were seen between the 139-group score and all nutritional biomarkers (25-hydroxyvitamin $\mathrm{D}\left(\mathrm{r}_{\mathrm{s}}=0.14\right)$, vitamin $\mathrm{C}\left(\mathrm{r}_{\mathrm{s}}=0.30\right)$, total carotenoids $\left(\mathrm{r}_{\mathrm{s}}=\right.$ $0.25)$, total serum folate $\left(r_{s}=0.42\right)$, homocysteine $\left(r_{s}=-0.25\right)$ and vitamin $\left.B 12\left(r_{s}=0.21\right)\right)$. Correlations with the 20-group score were only slightly attenuated.

The 20-group diet quality score showed reasonable agreement with the full 139-item score. Both scores were correlated with nutritional biomarkers. A short 20-item FFQ can provide a meaningful and easy-to-implement tool to assess diet quality in adolescents.

\section{Conflict of Interest}

There is no conflict of interest. 